\title{
miR-198 functions as a tumor suppressor in breast cancer by targeting CUB domain-containing protein 1
}

\author{
YINGBIN HU, ZIYUAN TANG, BONIAN JIANG, JUYING CHEN and ZHONGPIN FU \\ Department of Colorectal Surgery, The Affiliated Cancer Hospital of Xiangya School of Medicine, Central South University, \\ Changsha, Hunan 410013, P.R. China
}

Received June 27, 2015; Accepted August 5, 2016

DOI: $10.3892 / \mathrm{ol} .2017 .5673$

\begin{abstract}
The molecular mechanisms underlying the dysregulation of microRNAs (miRs) have been previously documented in breast cancer. miR-198 has been reported to be deregulated in several human cancers. However, the detailed effects of miR-198 on breast cancer progression remain unclear. Using quantitative polymerase chain reaction analysis, we demonstrated in the present study that miR-198 was downregulated in breast cancer tissues and cell lines, and that downregulation of miR-198 was significantly correlated with lymph node metastasis. Functional studies revealed that miR-198 inhibited cell proliferation and migration and promoted cell adhesion in aggressive breast cancer cells in vitro. In addition, we observed that CUB domain-containing protein 1 (CDCP1) was a direct target of miR-198, and that knockdown of CDCP1 inhibited cell proliferation and migration, and promoted cell adhesion, which was similar to the effects of overexpression of miR-198. Taken together, we provide evidence to characterize the role of miR-198/CDCP1 interaction in breast cancer, which may be useful in breast cancer therapy.
\end{abstract}

\section{Introduction}

Breast cancer is the most frequent cancer affecting females, and the leading cause of cancer-related mortality among females worldwide (1). Although the disease has been studied widely, its underlying molecular mechanisms have not been fully elucidated. microRNAs (miRNAs) are a class of non-coding small RNAs ( 22 nucleotides) which negatively regulate the expression of target messenger RNAs (mRNAs) by binding to their 3'-untranslated regions (3'UTRs), causing mRNA

Correspondence to: Dr Yingbin Hu, Department of Colorectal Surgery, The Affiliated Cancer Hospital of Xiangya School of Medicine, Central South University, 283 Tongzipo Road, Changsha, Hunan 410013, P.R. China

E-mail: huyingbin2014@163.com

Key words: miR-198, CUB domain-containing protein 1, breast cancer, cell proliferation, cell migration, cell adhesion degradation and/or translation inhibition (2). miRNAs have been implicated in a variety of biological processes, including embryonic development, cell differentiation and diseases including human cancer (3). miR-198 has been reported to be deregulated in several human cancers, including colorectal (4), lung (5), pancreatic $(6,7)$, hepatocellular $(8,9)$, prostate $(10)$ and esophageal cancer (11). However, the involvement and effects of miR-198 on breast cancer progression and the underlying mechanism remain unknown.

CUB domain-containing protein 1 (CDCP1) has been widely reported to be highly expressed in various human cancers, and is significantly correlated with tumor malignancy and poor prognosis (12-17). CDCP1 is a transmembrane protein with several conserved tyrosine residues in the cytoplasmic domain that may be phosphorylated by the Src family kinases $(18,19)$. Previous studies have indicated that CDCP1 is involved in tumorigenesis processes by regulating cell migration ability and matrix degradation in a tyrosine phosphorylation-dependent manner (17). In breast cancer, tumors with a high level of CDCP1 expression demonstrate higher levels of proliferation (20), and CDCP1 is also suggested to be responsible for the regulation of adhesion and motility in breast cancer cells (21). These results indicate that CDCP1 may play a critical role in human breast cancer progression. However, the molecular mechanisms underlying CDCP1 regulation in human breast cancer cells have not been fully elucidated. Our computational prediction revealed that the CDCP1 3'-untranslated region (3'UTR) has miR-198 binding sites, suggesting that CDCP1 may be a direct target of miR-198.

In the present study, we aimed to elucidate the involvement of the miR-198/CDCP1 interaction in human breast cancer. Our results indicated that miR-198 was frequently downregulated in human breast cancer tissues and cell lines. In addition, enhanced expression of miR-198 reduced cell proliferation and migration, and promoted cell adhesion in breast cancer cells in vitro. Moreover, transcription activator-like effector nuclease (TALEN)-based CDCP1 silencing inhibited cell proliferation and migration, and promoted cell adhesion, which was similar to the effects of overexpression of miR-198. Luciferase reporter assay further demonstrated that miR-198 directly targeted the 3'UTR of CDCP1. Thus, we provided evidence to characterize the role of miR-198 and CDCP1 in human breast cancer, which may be useful for effective clinical therapies in the future. 


\section{Materials and methods}

Tissue samples and cell lines. Forty-nine clinical breast tumor tissues and the adjacent tissues (at least $5 \mathrm{~cm}$ away from the primary tumor) were collected at Xiangya Hospital, Changsha, China. All patients signed an informed consent form and the study was approved by the Independent Ethical Committee of Central South University, Changsha, China. Samples were stored at $-80^{\circ} \mathrm{C}$ until use. Four aggressive breast cancer cell lines (MCF-7, MDA-MB-231, BT474 and BT549) and a normal breast cell line (MCF10A) were used. Cells were grown routinely in HEPES-buffered Dulbecco's modified Eagle's medium containing 10\% fetal bovine serum (Gibco Life Technologies, Carlsbad, CA, USA) and cultured under $5 \% \mathrm{CO}_{2}$ humidified air.

Reverse transcription-quantitative polymerase chain reaction $(R T-q P C R)$. Total RNAs were prepared using TRIzol reagent (Invitrogen Life Technologies, Carlsbad, CA, USA) according to the manufacturer's instructions. The extracted RNA was reverse-transcribed to cDNA using a PrimeScript reagent kit (Promega Corporation, Madison, WI, USA). The relative expression of CDCP1 mRNA was detected by SYBR-Green qPCR assay (Bio-Rad Laboratories, Inc., Hercules, CA, USA) performed on an ABI Prism 7700 (Applied Biosystems; Thermo Fisher Scientific, Inc., Waltham, MA, USA). $\beta$-actin was used as a control to normalize the starting quantity of RNA. The specific primers were as follows: CDCP1, F: 5'-TCT GCAAGGCTGTGACCAAG-3', R: 5'-GCTCATTACTCA AGTCAACCAC-3'; $\beta$-actin, F: 5'-AGGGGCCGGACTCGT CATACT-3', R: 5'-GGCGGCACCACCATGTACCCT-3'. The specific primers sets for miR-198, U6 and the PCR mix were purchased from GeneCopoeia, Inc. (Rockville, MD, USA). The expression of U6 was used as an endogenous control. Reactions for each sample were performed in triplicate. Relative expression levels were calculated using the $2^{-\Delta \Delta C t}$ method.

Western blot analysis. Total cellular extracts were prepared from each group of cells with $200 \mathrm{ml}$ lysis buffer and subjected to western blot analysis. Approximately $50 \mu \mathrm{g}$ total protein was separated by sodium dodecyl sulphate-polyacrylamide gel electrophoresis, transferred to a polyvinylidene fluoride membrane and incubated with the indicated antibodies, followed by horseradish peroxidase-conjugated secondary antibody. Signals were visualized using enhanced chemiluminescence (ECL) substrates (Millipore, Billerica, MA, USA). The protein bands were visualized using an ECL detection kit (GE Healthcare Life Sciences, Chalfont, UK) as recommended by the manufacturer. $\beta$-actin was used for normalization. Antibodies of CDCP1 and $\beta$-actin were obtained from Abzoom (Abzoom Biolabs, Dallas, TX, USA).

Dual luciferase reporter assay. A fragment of the 3'UTR of CDCP1 containing the predicted miR-198 target site was amplified and inserted into the psiCHECK-2 vector (Promega Corporation) downstream of the luciferase gene sequence. A psiCHECK-2 construct containing the 3'UTR of CDCP1 with a mutant sequence of miR-198 was synthesized. The wild-type 3'UTR of CDCP1 (Wt-3'UTR of CDCP1) and mutant 3'UTR of CDCP1 (Mut-3'UTR of CDCP1) primers were as follows: Wt-3'UTR of CDCP1, F: 5'-CTCGAGGCA
AGCCCTGGATTCAGAGT-3', R: 5'-GCGGCCGCGGAT AACCACGAACCGACCTA-3'; Mut-3'UTR of CDCP1, F: 5'-GCGGCCGCGCAAGCCCTGGATTCAGAGT-3', R: 5'-CTCGAGGGATAACCACGAACCGACCTA-3'. MCF-7 and MDA-MB-231 cells were plated in 96-well plates, then the Wt-3'UTR of CDCP1-psi-CHECK2 or the Mut-3'UTR of CDCP1-psi-CHECK2 was co-transfected with pre-miR-198 and pre-scramble mimics, respectively. The untreated group was used as a control. Luciferase activity was detected using a dual-luciferase reporter gene assay kit (Promega Corporation) and normalized to Renilla activity.

TALEN-mediated knockout of CDCP1. Loss of function is a powerful approach in the study of gene function. In this study, we used TALEN technology to knock out the CDCP1 gene in human breast cancer MCF-7 and MDA-MB-231 cells. TALENs designed to target CDCP1 gene were purchased from Sidansai Biotechnology (Shanghai, China). Cells in 24-well plates were transfected with $400 \mathrm{ng}$ TALEN expression plasmids using Lipofectamine 2000 (Invitrogen Life Technologies) according to the manufacturer's instructions. Western blot analysis was used to examine the $\mathrm{CDCP} 1$ protein expression to validate the efficiency of TALEN plasmids.

Lentiviral miR-198 infection. Lentiviruses containing miR-198 (Lv-miR-198) or scramble (Lv-scramble) were purchased from GeneChem (Shanghai, China). The MCF-7 and MDA-MB-231 cells were cultured to $60-70 \%$ of the plates, and then a concentration of $3 \times 10^{4} \mathrm{TU} /$ well Lv-miR-198 or Lv-scramble lentivirus was added. RT-qPCR and western blot analysis were performed to determinate the mRNA and protein levels of CDCP1 in the MCF-7 and MDA-MB-231 cells after being infected for 7 days. The cells stably infected with lentivirus were expanded and harvested for further analysis.

Cell proliferation assay. 3-[4,5-dimethylthiazol-2-yl]-2,5 diphenyl tetrazolium bromide (MTT) assay was performed to evaluate cell proliferation. Briefly, cells were allowed to grow in 96-well plates with 5,000 cells per well, and incubated for 24, 48 and $72 \mathrm{~h}$, then $10 \mathrm{mg} / \mathrm{ml}$ MTT was added to the cells and incubated for $1 \mathrm{~h}$. The reaction was then terminated by removal of the supernatant, and $200 \mu \mathrm{l}$ dimethyl sulfoxide was added. After $1 \mathrm{~h}$ of incubation, the optical density at $570 \mathrm{~nm}$ of each well was measured with a microplate reader (Bio-Rad Laboratories, Inc.).

Cell migration assay. Cell migration was determined by Transwell assay. Cells suspended in serum-free medium were added into the upper chamber of the insert with Matrigel. Following $24 \mathrm{~h}$ incubation at $37^{\circ} \mathrm{C}$, cells remaining on the upper side of the membrane were carefully removed, while cells that had migrated through the membrane were fixed with $75 \%$ alcohol and stained with crystal violet for $25 \mathrm{~min}$, then washed with water and dried in air. The imaging and counting of cell numbers were performed using an inverted microscope (Nikon Corporation, Tokyo, Japan).

Cell adhesion assay. For adhesion assay, cells were seeded in a Matrigel-coated 96-well-plate. Following incubation for $1 \mathrm{~h}$, the wells were washed twice with phosphate-buffered saline, 
Table I. Correlation of miR-198 expression with clinicopathological features of breast cancer tissues.

\begin{tabular}{|c|c|c|c|c|}
\hline \multirow[b]{2}{*}{ Clinicopathological features } & \multirow[b]{2}{*}{ Number of cases } & \multicolumn{2}{|c|}{ miR-198 expression } & \multirow[b]{2}{*}{ P-value } \\
\hline & & High, n (\%) & Low, n (\%) & \\
\hline Age (years) & & & & 0.878 \\
\hline$\leq 40$ & 7 & $2(28.6)$ & $5(71.4)$ & \\
\hline $40-50$ & 23 & $9(39.1)$ & $14(60.9)$ & \\
\hline $50-60$ & 13 & $6(46.2)$ & $7(53.8)$ & \\
\hline$\geq 60$ & 6 & $2(33.3)$ & $4(66.7)$ & \\
\hline TNM classification $(\mathrm{T})$ & & & & 0.971 \\
\hline $\mathrm{T} 1$ & 7 & $3(42.9)$ & $4(57.1)$ & \\
\hline $\mathrm{T} 2$ & 34 & $13(38.3)$ & $21(61.7)$ & \\
\hline $\mathrm{T} 3$ & 8 & $3(37.5)$ & $5(62.5)$ & \\
\hline TNM classification $(\mathrm{N})$ & & & & 0.036 \\
\hline No & 39 & $18(46.2)$ & $21(53.8)$ & \\
\hline N1 & 10 & $1(10.0)$ & $9(90.0)$ & \\
\hline
\end{tabular}

TNM, tumor-node-metastasis.

fixed in $4 \%$ paraformaldehyde and stained with crystal violet. The imaging and counting of adherent cells were performed using an inverted microscope (Nikon Corporation).

Statistical analysis. All data are presented as the mean values \pm standard deviation. Student's t-test was used to analyze the differences in the experiments. The Chi-squared test was used to demonstrate the differences in miR-198 or CDCP1 expression with clinicopathological features. $\mathrm{P}<0.05$ was considered to indicate a statistically significant difference.

\section{Results}

miR-198 is downregulated, while CDCP1 is upregulated, in human breast cancer tissues and cell lines. To examine the expression signature of miR-198 in human breast cancer progression, we first performed miRNA-based RT-qPCR analysis in 49 clinical tumor samples and matched adjacent tissues. As shown in Fig. 1A, we observed that the expression of miR-198 was significantly decreased in selected tumor tissues compared with the matched adjacent tissues. Correlation analysis of miR-198 expression with clinicopathological features revealed that downregulated miR-198 expression was significantly correlated with lymph node metastasis $(\mathrm{P}=0.036$, Table I). We then moved to breast cell lines, and observed that the expression of miR-198 was significantly lower in the four invasive breast cancer cell lines (BT474, MDA-MB-231, MCF-7 and BT549 cells) than in the normal breast cell line MCF10A (Fig. 1B). Thus, our data suggest a strong link between downregulation of miR-198 and the pathogenesis of breast cancer. Contrary to miR-198, we observed that CDCP1 was significantly upregulated in selected clinical tumor samples (Fig. 1C) and invasive breast cancer cell lines (Fig. 1D). We also analyzed the association of CDCP1 expression with clinicopathological parameters, and observed that high CDCP1 expression levels were correlated with lymph node metastasis
$(\mathrm{P}=0.028$, Table II). These data suggest that upregulation of CDCP1 may be involved in breast cancer progression.

miR-198 directly targets CDCP1 and inhibits its expression. The findings above, as well as the computational prediction (Fig. 2A) prompted us to further investigate whether miR-198 directly targets CDCP1. To do so, we cloned the wild-type 3'UTR (Wt-3'UTR) of CDCP1 containing the predicted binding site of miR-198 downstream of a luciferase reporter gene (Fig. 2A). We also constructed its mutant version (Mut-3'UTR of CDCP1) by binding site mutagenesis. The vectors were co-transfected with miR-198 mimics (pre-miR-198) or corresponding scrambled mimics (pre-scramble) as controls into MCF-7 and MDA-MB-231 cells, respectively. The luciferase activity of cells transfected with miR-198 mimic was significantly decreased compared with that of control cells (Fig. 2A). Additionally, the miR-198-mediated repression of luciferase activity was abolished by the mutant putative binding site (Fig. 2A). Furthermore, we tested the inhibitory effect of miR-198 on CDCP1 expression in MCF-7 and MDA-MB-231 cells. RT-qPCR and western blot analysis revealed that enhanced miR-198 significantly decreased CDCP1 mRNA and protein levels compared with cells transfected with control in MCF-7 and MDA-MB-231, respectively (Fig. 2B-D). Taken together, our results suggest that CDCP1 is a direct functional target of miR-198 in breast cancer cells.

miR-198 represses cell proliferation and migration and promotes cell adhesion in breast cancer cells. With the understanding that miR-198 is significantly downregulated in breast cancer tissues, we investigated whether miR-198 might serve as a tumor suppressor in breast cancer. We restored miR-198 expression in MCF-7 and MDA-MB-231 cells, which demonstrated a lower expression of miR-198 in the four selected breast cancer cell lines, by lentiviral infection with LV-miR-198 or 
Table II. Correlation of CDCP1 expression with clinicopathological features of breast cancer tissues.

CDCP1 expression

Clinicopathological features

Number of cases

High, n (\%)

Low, n (\%)

P-value

Age (years)

$\leq 40$

7

40-50

23

$50-60$

13

$\geq 60$

15 (65)

8 (62)

4 (67)

0.404

TNM classification (T)

$\mathrm{T} 1$

7

$5(71)$

22 (65)

T2

34

7 (88)

0.978

T3

8

TNM classification (N)

$\begin{array}{ll}\text { N0 } & 32 \\ \text { N1 } & 10 \\ \text { N2 } & 7\end{array}$

A

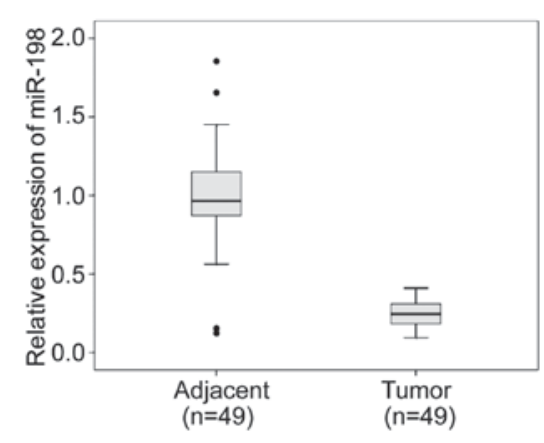

C

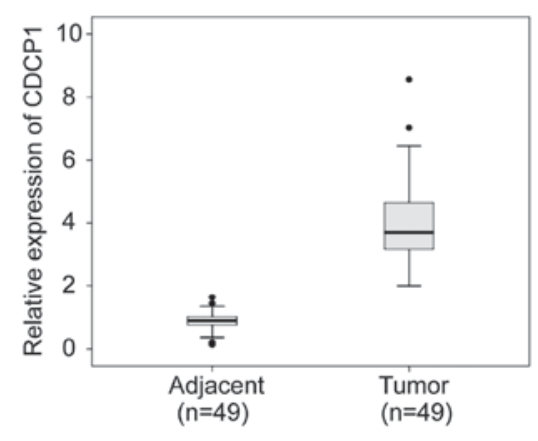

B

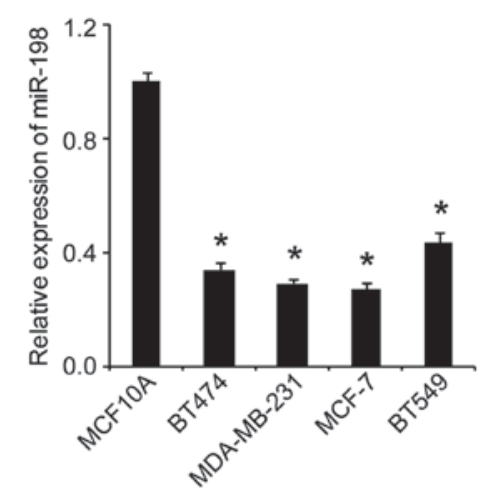

D

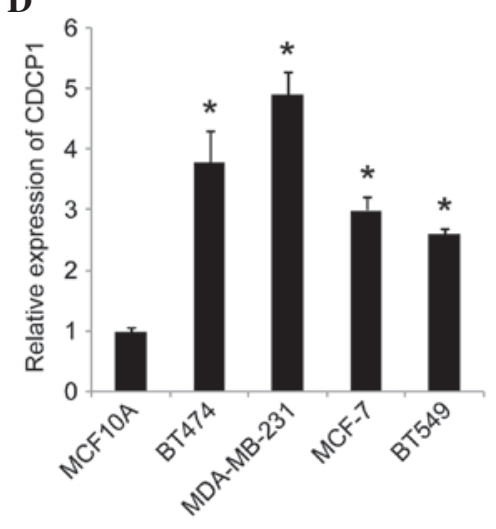

Figure 1. Expression of miR-198 and CUB domain-containing protein 1 (CDCP1) in human breast cancer tissues and cell lines. (A) Boxplots show the expression levels of miR-198 in 49 clinical breast tumor samples and matched adjacent tissues measured by reverse transcription-quantitative polymerase chain reaction (RT-qPCR). (B) Expression of miR-198 in the normal breast cell line (MCF10A) and four invasive breast cancer cell lines (BT474, MDA-MB-231, MCF-7 and BT549). (C) Boxplots show the expression levels of CDCP1 in the same 49 clinical breast tumor samples and matched adjacent tissues measured by RT-qPCR. (D) Expression of CDCP1 in the normal breast cell line and four invasive breast cancer cell lines. * P<0.05 vs. normal tissues or normal cell line; data shown are the means \pm standard deviation.

Lv-scramble lentivirus. RT-qPCR was performed to confirm that miR-198 was upregulated in MCF-7 and MDA-MB-231 cells following Lv-miR-198 infection (Fig. 3A). We then investigated the effect of miR-198 on cell proliferation, 


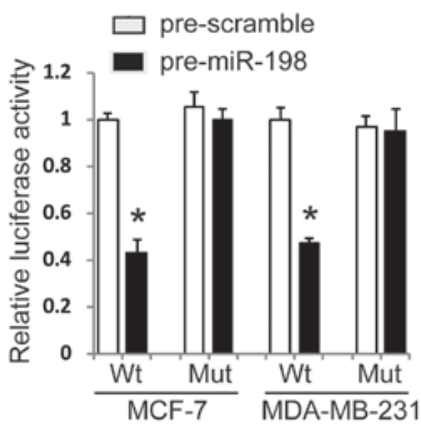

B

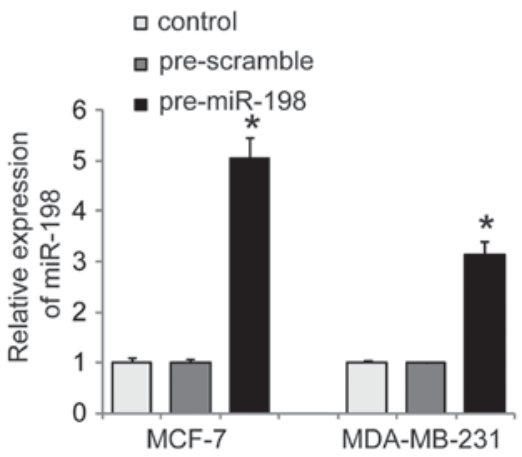

D

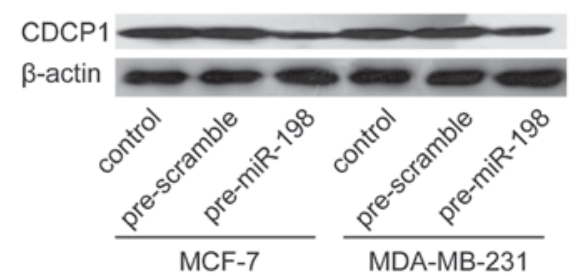

C a control
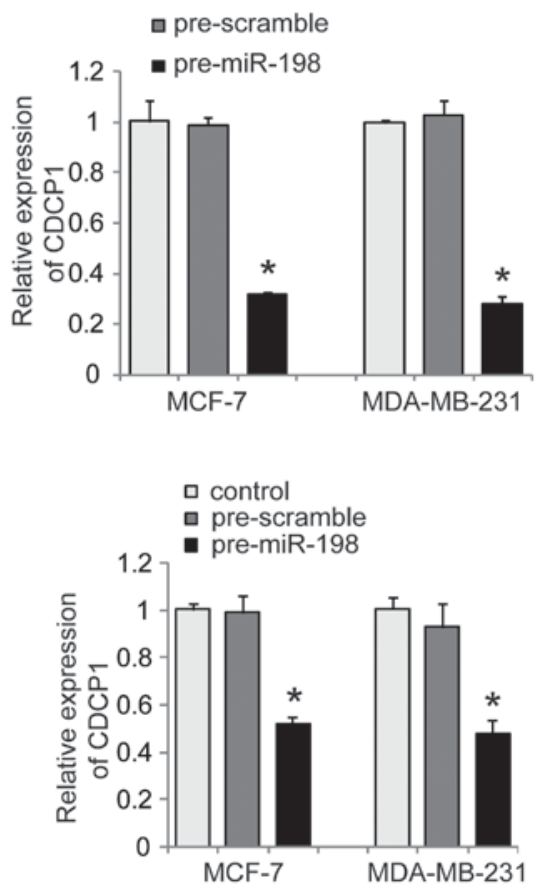

Figure 2. miR-198 directly targets CUB domain-containing protein 1 (CDCP1) and inhibits its expression. (A) Predicted binding sequences of miR-198 in the 3'-untranslated region (3'UTR) of CDCP1. The seed region of the 3'UTR of CDCP1 was mutated, and the fragment of wild-type (Wt) or mutant (Mut) 3'UTR of CDCP1 was subcloned into the luciferase reporter vector. Luciferase assay was performed in MCF-7 and MDA-MB-231 cells cotransfected with miR-198 mimics (pre-miR-198) or scrambled mimics (pre-scramble). The repression of luciferase activity by CDCP1 3'UTR was dependent on miR-198. Mutated CDCP1 3'UTR abrogated miR-198-mediated inhibition of luciferase activity. (B) Reverse transcription-quantitative polymerase chain reaction (RT-qPCR) was used to assess the expression of miR-198 following transfection with pre-miR-198, pre-scramble or control in MCF-7 and MDA-MB-231 cells, respectively (C and D) RT-qPCR and western blot analysis of CDCP1 expression following transfection with pre-miR-198, pre-scramble or control in MCF-7 and MDA-MB-231 cells, respectively; ${ }^{*} \mathrm{P}<0.05$ vs. control; data shown are the means \pm standard deviation.

migration and adhesion, respectively. The MTT assay revealed that overexpression of miR-198 inhibited the proliferation of MCF-7 and MDA-MB-231 cells (Fig. 4A). Transwell assay indicated that the enhanced expression of miR-198 could significantly inhibit cell migration ability compared with the control group in MCF-7 and MDA-MB-231 cells (Fig. 4B). Moreover, cell adhesion assays revealed that miR-198 notably promoted MCF-7 and MDA-MB-231 cell adhesion (Fig. 4C). Taken together, our findings suggest that miR-198 may play a suppressive role in breast cancer cell growth and migration.

Silencing of CDCP1 inhibits cell proliferation and migration, and promotes cell adhesion in breast cancer cells. To investigate the precise function of CDCP1 in breast cancer cells, we next silenced CDCP1 in breast cancer cells. We knocked out CDCP1 using TALEN technology, which represents a promising approach for targeted knockout of genes in cultured human cells (22). Western blot analysis was performed to confirm TALEN-mediated knockout efficiency in MCF-7 and MDA-MB-231 cells. As shown in Fig. 3B, the CDCP1 gene was silenced effectively in CDCP1-TALEN vector-transfected cells. Similar to miR-198 restoration, silencing CDCP1 by TALEN inhibited cell proliferation and migration ability (Fig. 4A and B), and promoted cell adhesion (Fig. 4C). We further knocked out CDCP1 in MCF-7 and MDA-MB-231 cells stably infected with LV-miR-198. As expected, a combination of silencing CDCP1 by TALEN and restored miR-198 had a more enhanced inhibitory effect on proliferation and migration ability than either silencing CDCP1 by TALEN or restoring miR-198 alone (Fig. 4A and B). Cell adhesion assays also revealed that cell adhesion was further promoted by the combinational treatment (Fig. 4C). Collectively, our results suggest that CDCP1 is involved in cell growth and migration of breast cancer cells. 
A

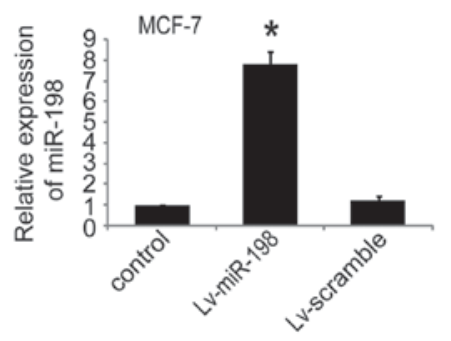

B

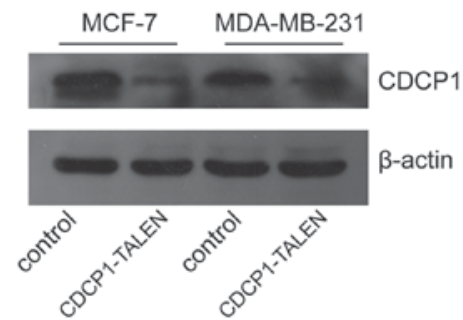

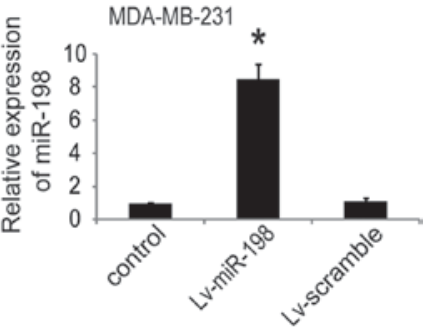

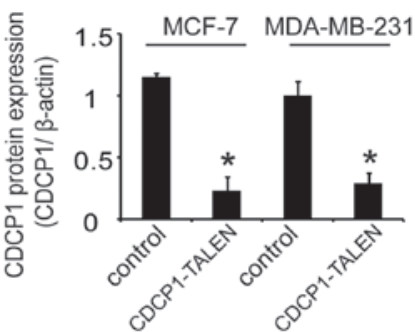

Figure 3. Validation of miR-198 overexpression and CUB domain-containing protein 1 (CDCP1) knockout in MCF-7 and MDA-MB-231 cells. (A) Reverse transcription-quantitative polymerase chain reaction was performed to confirm that miR-198 was upregulated in MCF-7 and MDA-MB-231 cells following Lv-miR-198 infection. (B) Following knockout using transcription activator-like effector nuclease (TALEN) technology in MCF-7 and MDA-MB-231 cells, CDCP1 expression was determined by western blot analysis and quantification to confirm TALEN-mediated knockout efficiency. "P<0.05 vs. control; data shown are the means \pm standard deviation.

A

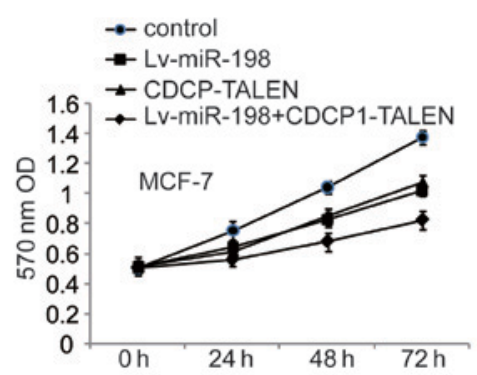

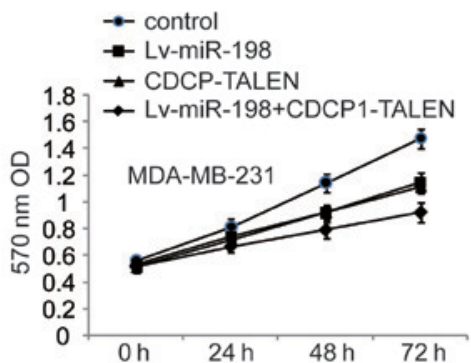

B

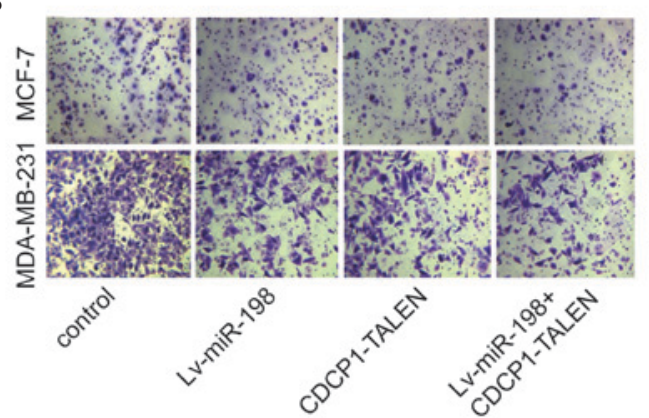

C

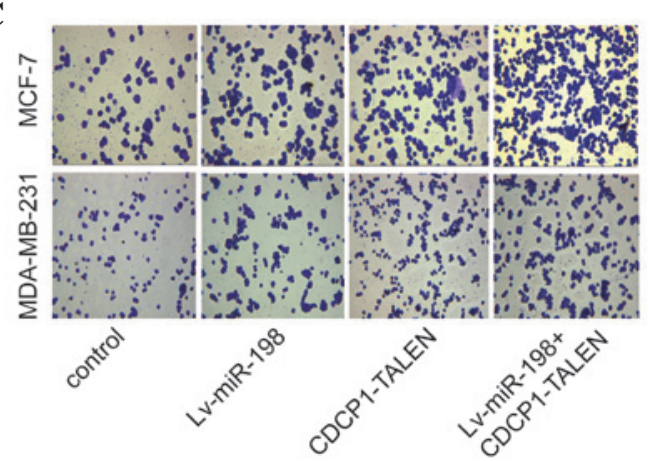

- control

- Lv-miR-198

- CDCP1-TALEN

- Lv-miR-198+CDCP1-TALEN

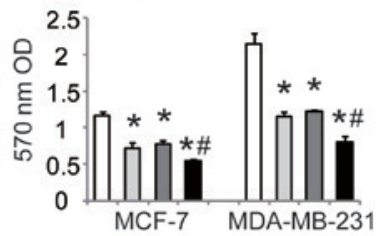

a control

- Lv-miR-198

LV-miR-198+CDCP1-TALEN

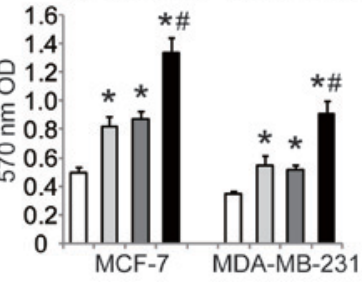

Figure 4. Effects of miR-198 overexpression and CUB domain-containing protein 1 (CDCP1) knockout in MCF-7 and MDA-MB-231 cells. (A) Cell proliferation was determined using 3-[4,5-dimethylthiazol-2-yl]-2,5 diphenyl tetrazolium bromide (MTT) assay. (B) Transwell assay was used to determinate cell migration. Representative images of crystal violet staining are shown (magnification, x100). (C) Cell adhesion assay was used to determinate cell adhesion. Representative images of crystal violet staining are shown (magnification, $\mathrm{x} 100$ ). ${ }^{*} \mathrm{P}<0.05$ vs. control, ${ }^{\#} \mathrm{P}<0.05$ vs. pre-miR-198 groups or CDCP1-transcription activator-like effector nuclease (TALEN) groups; data shown are the means \pm standard deviation. 


\section{Discussion}

In this study, we observed that miR-198 was downregulated in breast cancer tissues and cell lines compared with normal cancer tissues and normal cell lines. Then, we demonstrated that enforced miR-198 inhibited cell proliferation and migration of breast cancer cells, suggesting that miR-198 may function as a tumor suppressor in breast cancer metastasis. We also demonstrated that CDCP1 was upregulated in breast cancer tissues and invasive cell lines, and was a direct functional target of miR-198. Loss of function of CDCP1 through TALEN-based knockout suppressed cell proliferation and migration of breast cancer cells in vitro. Thus, we reasonably speculate that low expression of miR-198 contributes to CDCP1-mediated cell growth and migration in breast cancer cells.

miRNAs are frequently dysregulated in various cancers. Generally, miRNAs affect cancer development through post-transcriptional regulation of their target genes (23). Thus, the miRNA/target link in certain cancers may shed light on the molecular mechanism underlying cancer progression and provide useful potential therapeutic targets for the clinical treatment of certain cancers. miR-198 was reported to be located in the 3'UTR of follistatin-like 1 messenger RNA, which promotes keratinocyte migration, whereas miR-198 expression has the opposite effect (24). In human cancers, miR-198 was reported to be downregulated in colorectal (4), lung (5), pancreatic (6) and hepatocellular carcinoma $(8,9)$, and generally acts as a tumor suppressor by inhibiting cancer cell growth and migration. In contrast, high expression of miR-198 was noted to be associated with a shorter disease-free survival and overall survival time in pancreatic ductal adenocarcinomas (7), with poor prognosis in esophageal cancer (11) and in high-grade prostate tumors (10). These data suggest that the roles of miR-198 may vary in different types of cancer. In the present study, we demonstrated that miR-198 exerted inhibitory effects on the proliferation and migration of breast cancer cells.

CDCP1 is an integral membrane protein whose expression is frequently upregulated and positively correlated with poor prognosis in various types of cancer. CDCP1 was identified as a protein functionally involved in cancer metastasis in 2003 (13), and was recently shown to promote migration and peritoneal dissemination of gastric scirrhous carcinoma (25), and be a unique target gene of hypoxia-inducible factor $2 \alpha$ involved in the regulation of renal cancer metastasis (26). In human pancreatic cancers, CDCP1 was also observed to be a prognostic factor, regulating cell migration and extracellular matrix degradation (16). In breast cancer, previous studies suggest that there is a positive correlation between poor prognosis and high expression of CDCP1 in tumor tissues $(13,20)$. In the present study, we confirmed that CDCP1 was upregulated in breast cancer tissues and invasive cell lines. Using MDA-MB-231 and MCF-7 breast cancer cells as in vitro models, as well as silencing CDCP1 using TALEN technology, we observed that CDCP1 is closely associated with cell proliferation, migration and adhesion in breast cancer cells. Combined with a previous observation that antibody-mediated CDCP1 degradation significantly inhibited tumor growth in a mouse xenograft model in vivo (27), we suggest that high expression of CDCP1 contributes to migration in breast cancer cells and may be involved in human breast tumorigenesis.

In conclusion, we have established a new miR-198/CDCP1 link in breast cancer cells, in which the loss of miR-198 may result in gained expression of CDCP1, which endows breast cancer cells with improved migration capacity. The restoration of miR-198 and/or inhibition of CDCP1 expression may be a promising strategy for breast cancer therapy.

\section{References}

1. Siegel R, Naishadham D and Jemal A: Cancer statistics, 2013. CA Cancer J Clin 63: 11-30, 2013.

2. Bartel DP: MicroRNAs: genomics, biogenesis, mechanism, and function. Cell 116: 281-297, 2004.

3. Sayed D and Abdellatif M: MicroRNAs in development and disease. Physiol Rev 91: 827-887, 2011.

4. Wang M, Wang J, Kong X, Chen H, Wang Y, Qin M, Lin Y, Chen H, Xu J, Hong J, et al: miR-198 represses tumor growth and metastasis in colorectal cancer by targeting fucosyl transferase 8 . Sci Rep 4: 6145, 2014.

5. Yang J, Zhao H, Xin Y and Fan L: MicroRNA-198 inhibits proliferation and induces apoptosis of lung cancer cells via targeting FGFR1. J Cell Biochem 115: 987-995, 2014.

6. Marin-Muller C, Li D, Bharadwaj U, Li M, Chen C, Hodges SE, Fisher WE, Mo Q, Hung MC and Yao Q: A tumorigenic factor interactome connected through tumor suppressor microRNA-198 in human pancreatic cancer. Clin Cancer Res 19: 5901-5913, 2013.

7. Vychytilova-Faltejskova P, Kiss I, Klusova S, Klusova S, Hlavsa J, Prochazka V, Kala Z, Mazanec J, Hausnerova J, Kren L, et al: MiR-21, miR-34a, miR-198 and miR-217 as diagnostic and prognostic biomarkers for chronic pancreatitis and pancreatic ductal adenocarcinoma. Diagn Pathol 10: 38, 2015.

8. Elfimova N, Sievers E, Eischeid H, Kwiecinski M, Noetel A, Hunt H, Becker D, Frommolt P, Quasdorff M, Steffen HM, et al: Control of mitogenic and motogenic pathways by miR-198, diminishing hepatoma cell growth and migration. Biochim Biophys Acta 1833: 1190-1198, 2013.

9. Tan S, Li R, Ding K, Lobie PE and Zhu T: miR-198 inhibits migration and invasion of hepatocellular carcinoma cells by targeting the HGF/c-MET pathway. FEBS Lett 585: 2229-2234, 2011.

10. Walter BA, Valera VA, Pinto PA and Merino MJ: Comprehensive microRNA profiling of prostate cancer. J Cancer 4: 350-357, 2013.

11. Qi B, Yao WJ, Zhao BS, Qin XG, Wang Y, Wang WJ, Wang TY, Liu SG and Li HC: Involvement of microRNA-198 overexpression in the poor prognosis of esophageal cancer. Asian Pac J Cancer Prev 14: 5073-5076, 2013.

12. Awakura Y, Nakamura E, Takahashi T, Kotani H, Mikami Y, Kadowaki T, Myoumoto A, Akiyama H, Ito N, Kamoto T, et al: Microarray-based identification of CUB-domain containing protein 1 as a potential prognostic marker in conventional renal cell carcinoma. J Cancer Res Clin Oncol 134: 1363-1369, 2008.

13. Hooper JD, Zijlstra A, Aimes RT, Liang H, Claassen GF, Tarin D, Testa JE and Quigley JP: Subtractive immunization using highly metastatic human tumor cells identifies SIMA135/CDCP1, a $135 \mathrm{kDa}$ cell surface phosphorylated glycoprotein antigen. Oncogene 22: 1783-1794, 2003.

14. Ikeda J, Oda T, Inoue M, Uekita T, Sakai R, Okumura M, Aozasa K and Morii E: Expression of CUB domain containing protein (CDCP1) is correlated with prognosis and survival of patients with adenocarcinoma of lung. Cancer Sci 100: 429-433, 2009.

15. Liu H, Ong SE, Badu-Nkansah K, Schindler J, White FM and Hynes RO: CUB-domain-containing protein 1 (CDCP1) activates Src to promote melanoma metastasis. Proc Natl Acad Sci USA 108: 1379-1384, 2011.

16. Miyazawa Y, Uekita T, Hiraoka N, Fujii S, Kosuge T, Kanai Y, Nojima $Y$ and Sakai R: CUB domain-containing protein 1, a prognostic factor for human pancreatic cancers, promotes cell migration and extracellular matrix degradation. Cancer Res 70: 5136-5146, 2010

17. Uekita T and Sakai R: Roles of CUB domain-containing protein 1 signaling in cancer invasion and metastasis. Cancer Sci 102: 1943-1948, 2011 
18. Benes CH, Wu N, Elia AE, Dharia T, Cantley LC and Soltoff SP: The C2 domain of PKCdelta is a phosphotyrosine binding domain. Cell 121: 271-280, 2005.

19. Uekita T, Jia L, Narisawa-Saito M, Yokota J, Kiyono T and Sakai R: CUB domain-containing protein 1 is a novel regulator of anoikis resistance in lung adenocarcinoma. Mol Cell Biol 27: 7649-7660, 2007.

20. Ikeda JI, Morii E, Kimura H, Tomita Y, Takakuwa T,Hasegawa JI, Kim YK, Miyoshi Y, Noguchi S, Nishida T and Aozasa K: Epigenetic regulation of the expression of the novel stem cell marker CDCP1 in cancer cells. J Pathol 210: 75-84, 2006.

21. Seidel J, Kunc K, Possinger K, Jehn C and Lüftner D: Effect of the tyrosine kinase inhibitor lapatinib on CUB-domain containing protein (CDCP1)-mediated breast cancer cell survival and migration. Biochem Biophys Res Commun 414: 226-232, 2011

22. Cermak T, Doyle EL, Christian M, Wang L, Zhang Y, Schmidt C, Baller JA, Somia NV, Bogdanove AJ and Voytas DF: Efficient design and assembly of custom TALEN and other TAL effector-based constructs for DNA targeting. Nucleic Acids Res 39: e82, 2011.
23. Garzon R, Marcucci G and Croce CM: Targeting microRNAs in cancer: rationale, strategies and challenges. Nat Rev Drug Discov 9: 775-789, 2010.

24. Sundaram GM, Common JE, Gopal FE, Srikanta S, Lakshman K, Lunny DP, Lim TC, Tanavde V, Lane EB and Sampath P: 'See-saw' expression of microRNA-198 and FSTL1 from a single transcript in wound healing. Nature 495: 103-106, 2013.

25. Uekita T, Tanaka M, Takigahira M, Miyazawa Y, Nakanishi Y, Kanai Y, Yanagihara K and Sakai R: CUB-domain-containing protein 1 regulates peritoneal dissemination of gastric scirrhous carcinoma. Am J Pathol 172: 1729-1739, 2008

26. Emerling BM, Benes CH, Poulogiannis G, Bell EL, Courtney K, Liu H, Choo-Wing R, Bellinger G, Tsukazawa KS, Brown V, et al: Identification of CDCP1 as a hypoxia-inducible factor $2 \alpha$ (HIF-2 $\alpha$ ) target gene that is associated with survival in clear cell renal cell carcinoma patients. Proc Natl Acad Sci USA 110: 3483-3488, 2013.

27. Kollmorgen G, Niederfellner G, Lifke A, Spohn GJ, Rieder N, Harring SV, Bauss F, Burtscher H, Lammers R and Bossenmaier B: Antibody mediated CDCP1 degradation as mode of action for cancer targeted therapy. Mol Oncol 7: 1142-1151, 2013. 